Article

\title{
Synthesis and Characterization of New Thiazolidinones Containing Coumarin Moieties and Their Antibacterial and Antioxidant Activities
}

Naceur Hamdi ${ }^{1, *}$, Abdullah Sulaiman Al-Ayed ${ }^{1}$, Ridha Ben Said ${ }^{1}$ and Alary Fabienne ${ }^{2}$

1 College of Science and Arts at Ar-Rass, Qassim University, P.O. Box 53, Saudi Arabia

2 Laboratoire de chimie et physique quantiques, Université Paul Sabatier-Bât. 3R1b4, 118 route de Narbonne, 31062 Toulouse Cedex 09, France

* Author to whom correspondence should be addressed; E-Mail: naceur.hamdi@isste.rnu.tn; Tel.: +966-505-142-736; Fax: +966-6333-9351.

Received: 18 June 2012; in revised form: 21 July 2012 / Accepted: 24 July 2012 /

Published: 3 August 2012

\begin{abstract}
New coumarin derivatives, namely (2-(4-methyl-2-oxo- $2 H$-chromen-7-yloxy)$N$-(4-oxo-2-phenylthiazolidin-3-yl)acetamide, $\quad N$-(2-(3-methoxyphenyl)-4-oxothiazolidin3-yl)-2-(4-methyl-2-oxo-2H-chromen-7-yloxy)acetamide, 2-(4-methyl-2-oxo-2H-chromen7-yloxy)- $N$-(4-oxo-2-(2,3,4trimethoxyphenyl)thiazolidin-3-yl)acetamide and $\mathrm{N}$-(2-(4bromophenyl)-4-oxothiazolidin-3-yl)-2-(4-methyl-2-oxo-2H-chromen-7-yloxy)acetamide) were synthesized starting from 4-methyl-7-hydroxycoumarin. The structures of the obtained compounds were confirmed by analytical IR and NMR spectra to elucidate the different positions of protons and carbons and as well as theoretical studies (DFT/B3LYP). The new compounds were screened for antibacterial activity. Most of them are more active against $E$. coli $S$. aureus and B. subtilis than standard references.
\end{abstract}

Keywords: coumarin; ethyl bromoacetate; thiazolidinones; DFT studies; antibacterial activities

\section{Introduction}

Small ring heterocycles containing nitrogen and sulfur have been under investigation for a long time because of their important medicinal properties. Among the wide range of heterocycles explored to develop pharmaceutically important molecules, thiazoles have played an important role in medicinal 
chemistry. A survey of literature has shown that compounds having a thiazole nucleus possess a broad range of biological activities such as anti-inflammatory [1], antibacterial [2] and antifungal properties [3]. Among these type of molecules 4-thiazolidinones have shown to have various important biological activities such as antibacterial, antifungal, antiviral, diuretic, antituberculostatic, anti-HIV, antihistaminic, anticancer, anticonvulsant, anti-inflammatory and analgesic properties [4-11]. Recently, a study reported the synthesis, chemical and wide rang biological properties of a series of 4-thiazolidinone molecules [12-18]. Some of these compounds showed moderate to good biological properties. The observed interesting biological properties of this class of compounds impelled us to synthesize new examples with possible improved biological properties with applicative possibilities.

In the current study we aimed to synthesize some new coumarins derived from umbelliferone (7-hydroxycoumarin), with predictable biological activities. The chemical structures of the synthesized compounds were proven by IR, NMR spectra and elemental analysis data.

\section{Results and Discussion}

The starting compound (7-hydroxy-2-oxo-2H-chromen-4-yl)-acetic acid methyl ester (2) was prepared in $92 \%$ yield by esterification of 7-hydroxy-4-methylcoumarin (1). Hydrazinolysis of 2 with $86 \%$ hydrazine hydrate in ethanol at reflux for $4 \mathrm{~h}$ afforded hydrazide 3 in good yield. The FT-IR spectra of carbohydrazide 3 showed absorption bands at $3317 \mathrm{~cm}^{-1}$, possibly due to the hydrazide $\mathrm{NH}-\mathrm{NH}_{2}, 3269 \mathrm{~cm}^{-1}$ (aromatic $\left.\mathrm{CH}\right), 1711 \mathrm{~cm}^{-1}(-\mathrm{C}=\mathrm{O}$ carbonyl stretching) and in the range 1621-1640 $\mathrm{cm}^{-1}$ (-CO-NH-NH 2 groups). The ${ }^{1} \mathrm{H}-\mathrm{NMR}$ spectrum exhibited a singlet due to the $-\mathrm{CO}-\mathrm{N} H-\mathrm{NH}_{2}$ proton at $\delta 4.34 \mathrm{ppm}$. Methylene protons $\left(-\mathrm{OCH}_{2}\right)$ resonate as singlets at $4.94 \mathrm{ppm}$.

Refluxing 3 and aromatic aldehydes with a catalytic amount of glacial acetic acid in absolute ethanol for $4 \mathrm{~h}$ afforded the new series of coumarin $N^{\prime}$-benzylidene-2-(4-methyl-2-oxo- $2 \mathrm{H}$-chromen-7yloxy) acetohydrazide Schiff bases $\mathbf{4 a - d}$. The structures of compounds $\mathbf{4 a - d}$ were inferred from their analytical and spectroscopic properties. Thus the IR spectrum of compound 4 a showed characteristic bands in the range $1610 \mathrm{~cm}^{-1}(\mathrm{CONH})$, at $1681 \mathrm{~cm}^{-1}(\mathrm{C}=\mathrm{O}$, lactone $)$, and at $1258 \mathrm{~cm}^{-1}(-\mathrm{HC}=\mathrm{N}-$ azomethine). The ${ }^{1} \mathrm{H}-\mathrm{NMR}$ spectrum did not only show the absence of $\mathrm{NH}_{2}$ protons at 3.38 , but also the presence of the $\mathrm{N}=\mathrm{CH}$ proton at $8.24 \mathrm{ppm}$.

$\mathrm{N}$-(2-aryl-4-oxo-thiazolidin-3-yl)-2-(4-(2-aryl-4-oxo-thiazolidin-3-ylcarbamoyl)-methyl)-2-oxo-2 $\mathrm{H}$ chromen-7-yloxy)-acetamides $\mathbf{5 a - d}$ were obtained by reaction of the compounds $\mathbf{4 a - d}$ with thioglycolic acid in refluxing 1,4-dioxane for 6-8 h in the presence of anhydrous $\mathrm{ZnCl}_{2}$ (Scheme 1).

The IR spectrum of $\mathbf{5 b}$ showed a characteristic band at $1728 \mathrm{~cm}^{-1}$ that supports the presence in the molecule of a thiazolidinone $\mathrm{C}=\mathrm{O}$ group. The ${ }^{1} \mathrm{H}-\mathrm{NMR}$ spectrum of $\mathbf{5 b}$ displayed signals between $7.30-7.60 \delta \mathrm{ppm}$ for aromatic protons and a doublet at $4.84 \mathrm{ppm}$ ascribable to the thiazolidinones $\mathrm{CH}_{2}$ protons. The proposed 5a-d structures were evaluated by using a DFT/B3LYP approach implemented in the Gaussian 09 series programs [19]. The B3LYP hybrid functional has been used in describing potential energy surfaces (PES). The geometries of the compounds were fully optimized using analytic gradients. The harmonic vibrational frequencies of the stationary points of the PES have been calculated at the same level of theory in order to identify the local minima as well as to estimate the corresponding zero point vibrational energy (ZPE) [20]. For each atom no pseudopotential are used. A 
Def2-SVP EMSL Basis Set Exchange was employed for each atom [21]. Values of selected geometrical parameters are listed in Table 1 and optimized geometry for $\mathbf{5 a}$ is depicted in Figure 1.

Scheme 1. Synthesis of 2-(4-methyl-2-oxo- $2 H$-chromen-7-yloxy)- $N$-(4-oxo-2-arylthiazolidin3-yl) acetamide 5.<smiles>Cc1cc(=O)oc2cc(O)ccc12</smiles><smiles>CCOC(=O)CBr</smiles><smiles>[R]C(=O)OCCC(=O)OC(C)=O</smiles>
reflux $12 \mathrm{~h}$<smiles>C/C=N/NC(=O)COc1ccc2c(C)cc(=O)oc2c1</smiles>
4<smiles>CCOC(=O)COc1ccc2c(C)cc(=O)oc2c1</smiles>
$\mathrm{N}_{2} \mathrm{H}_{2}$, Ethanol reflux $4 \mathrm{~h}$<smiles>Cc1cc(=O)oc2cc(OCC(=O)NN)ccc12</smiles><smiles>Cc1cc(=O)oc2cc(OCC(=O)NN3C(=O)CSC3C)ccc12</smiles>

5

\begin{tabular}{ccc}
\hline Compounds 5 & $\mathbf{A r}$ & Yield \\
\hline $5 \mathrm{a}$ & $\mathrm{C}_{6} \mathrm{H}_{5}$ & 40 \\
$5 \mathrm{~b}$ & $p-\mathrm{OCH}_{3} \mathrm{C}_{6} \mathrm{H}_{4}$ & 76 \\
$5 \mathrm{c}$ & $2,3,4 \mathrm{OCH}_{3} \mathrm{C}_{6} \mathrm{H}_{2}$ & 52 \\
$5 \mathrm{~d}$ & $p-\mathrm{Br} \mathrm{C}_{6} \mathrm{H}_{4}$ & 72 \\
\hline
\end{tabular}


Table 1. DFT/B3LYP optimized geometrical parameters ${ }^{\text {a }}$ for $\mathbf{5 a}-\mathbf{d}$.

\begin{tabular}{|c|c|c|c|c|}
\hline Compounds & $5 \mathbf{a}$ & $5 \mathbf{b}$ & $5 \mathrm{c}$ & $5 d$ \\
\hline $1-2$ & 1,385 & 1.384 & 1.383 & 1.386 \\
\hline $1-12$ & 1.374 & 1.374 & 1.372 & 1.374 \\
\hline $1-5$ & 1.463 & 1.465 & 1.462 & 1.462 \\
\hline $2-3$ & 1.520 & 1.521 & 1.520 & 1.520 \\
\hline $3-4$ & 1.829 & 1.829 & 1.826 & 1.829 \\
\hline $4-5$ & 1.863 & 1.864 & 1.866 & 1.863 \\
\hline $5-6$ & 1.510 & 1.506 & 1.509 & 1.510 \\
\hline $12-13$ & 1.394 & 1.393 & 1.389 & 1.394 \\
\hline $13-14$ & 1,530 & 1.530 & 1.531 & 1.529 \\
\hline $14-15$ & 1.401 & 1.401 & 1.402 & 1.401 \\
\hline $15-7^{\prime}$ & 1.352 & 1.352 & 1.352 & 1.353 \\
\hline $1^{\prime}-2^{\prime}$ & 1.396 & 1.396 & 1.396 & 1.397 \\
\hline $1^{\prime}-9^{\prime}$ & 1.354 & 1.354 & 1.354 & 1.354 \\
\hline $2^{\prime}-3^{\prime}$ & 1.457 & 1.457 & 1.457 & 1.457 \\
\hline $3^{\prime}-4^{\prime}$ & 1.362 & 1.362 & 1.362 & 1.362 \\
\hline $4^{\prime}-10^{\prime}$ & 1.454 & 1.454 & 1.454 & 1.454 \\
\hline $5-\mathrm{H} 5$ & 1.105 & 1.106 & 1.101 & 1.105 \\
\hline 12-H12 & 1.020 & 1.020 & 1.020 & 1.020 \\
\hline 14-H14 & 1.107 & 1.108 & 1.108 & 1.108 \\
\hline $1-2-3$ & 111.1 & 111.0 & 110.7 & 111.1 \\
\hline $2-3-4$ & 107.8 & 107.8 & 107.6 & 107.8 \\
\hline $3-4-5$ & 92.9 & 92.8 & 93.0 & 92.9 \\
\hline $4-5-1$ & 103.4 & 103.2 & 103.4 & 103.5 \\
\hline $1-5-6$ & 115.5 & 115.6 & 115.6 & 115.5 \\
\hline $1-5-\mathrm{H} 5$ & 109.5 & 109.5 & 108.4 & 109.6 \\
\hline $2-1-12$ & 118.1 & 118.2 & 119.5 & 118.1 \\
\hline 1-12-H12 & 114.9 & 114.9 & 114.7 & 114.8 \\
\hline $1-12-13$ & 119,0 & 118.9 & 121.2 & 119.0 \\
\hline $12-13-14$ & 111.9 & 111.9 & 111.2 & 112.0 \\
\hline $13-14-15$ & 108.3 & 108.3 & 108.9 & 108.4 \\
\hline $1^{\prime}-2^{\prime}-3^{\prime}$ & 115.4 & 115.4 & 115.4 & 115.4 \\
\hline $2^{\prime}-3^{\prime}-4^{\prime}$ & 123.3 & 123.3 & 123.3 & 123.3 \\
\hline $3^{\prime}-4^{\prime}-10^{\prime}$ & 118.6 & 118.6 & 118.5 & 118.5 \\
\hline $4^{\prime}-10^{\prime}-9^{\prime}$ & 118.0 & 118.0 & 117.9 & 118.0 \\
\hline $1-2-3-4$ & 6.1 & 7.5 & -13.9 & 6.5 \\
\hline $2-3-4-5$ & -15.4 & -17.0 & 18.6 & -15.8 \\
\hline $3-4-5-1$ & 19.9 & 21.1 & -17.9 & 20.0 \\
\hline H5-5-6-11 & -12.6 & -11.8 & -0.7 & -12.8 \\
\hline $3-4-5-6$ & 144.3 & 145.8 & 109.4 & 144.5 \\
\hline $1-5-6-7$ & -136.1 & -135.1 & -122.1 & -136.4 \\
\hline $2-3-5-12$ & 1.0 & 1.1 & -0.9 & -11.6 \\
\hline $2-5-7^{\prime}-6^{\prime}$ & 113.0 & 114.1 & 109.1 & 118.2 \\
\hline $12-13-14-15$ & -158.1 & -157.1 & -161.7 & -155.2 \\
\hline $13-14-15-7^{\prime}$ & -175.5 & -174.8 & -177.7 & -174.9 \\
\hline
\end{tabular}


Table 1. Cont.

\begin{tabular}{lllll}
\hline Compounds & $\mathbf{5 a}$ & $\mathbf{5 b}$ & $\mathbf{5 c}$ & $\mathbf{5 d}$ \\
\hline $14-15-7^{\prime}-6^{\prime}$ & 178.5 & 178.2 & 176.8 & 179.2 \\
$7^{\prime}-8^{\prime}-9^{\prime}-10^{\prime}$ & 0.0 & 0.0 & 0.4 & 0.1 \\
$10^{\prime}-9^{\prime}-11^{\prime}-2^{\prime}$ & 0.0 & 0.1 & 0.0 & 0.0 \\
$1^{\prime}-2^{\prime}-3^{\prime}-4^{\prime}$ & 0.1 & 0.2 & 0.6 & 0.1 \\
\hline
\end{tabular}

${ }^{\mathrm{a}}$ Distances are in $\mathrm{A}^{\circ}$ and angles in degrees.

Figure 1. Optimized geometry for $\mathbf{5 a}$.

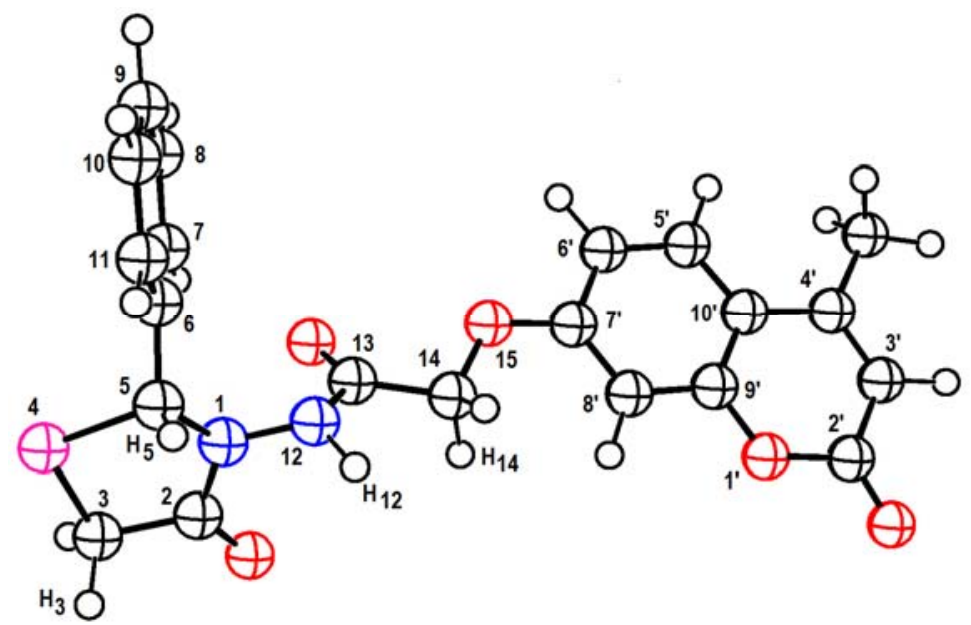

Some conclusions can be extracted from the results obtained. The calculated values for length and angles are similar for compounds 5a-d. This fact suggests that the $\mathrm{R}$ group has no influence on interatomic distances and angles. Additionally the dihedral angles 1-2-3-4, 2-3-4-5 and 3-4-5-1 indicate that the thiazolidinone ring in the compounds is not in a plane and that fact is not dependent on the $\mathrm{R}$ group. The plane constituted by the thia-ring is perpendicular to that of the coumarin group (for exemple the angle $2-5-6^{\prime}-7^{\prime}=113.0$ in 5a). It is important to point out that the $\mathbf{5 c}$ thia-ring displays a different orientation that those for the other calculated compounds. Important information on the chemical groups present in compounds $\mathbf{5 a}-\mathbf{d}$ can be obtained from their calculated vibrational spectra. As example, intensities and their harmonic wave numbers calculated with the method DFT/B3LYP and the corresponding experimental values of the compound $\mathbf{5 c}$ are grouped in Table 2.

Table 2. Theoretical and experimental vibrational frequencies $\left(\mathrm{cm}^{-1}\right)$ and theoretical infrared intensities $\left(\mathrm{km} \mathrm{mol}^{-1}\right)$ of $\mathbf{5 c}$.

\begin{tabular}{cccccc}
\hline & \multicolumn{2}{c}{ Experimental } & & \multicolumn{2}{c}{ Theoretical } \\
\cline { 2 - 3 } \cline { 5 - 5 } Functional group & Frequencies & Intensities & & Frequencies & Intensities \\
\hline CO thiaz. & 1666 & strong & & 1831 & 328 \\
CO lactone & 1682 & strong very & & 1862 & 652 \\
CO amide & 1712 & mean & & 1871 & 130 \\
NH & 3313 & weak & & 3513 & 23 \\
\hline
\end{tabular}

The values of the theoretical IR frequencies are in agreement with the experimental ones. The largest shifting is less than $10 \%$ that could be due to the approximations used in the DFT/B3LYP method; consequently the proposed structures were supported by theoretical calculations. 
The NBO analysis allows the determination of the atomic natural charges. Table 3 reports the natural charges of the acid protons in compound $\mathbf{5 c}$ calculated at DFT/B3LYP level of theory. We find that proton $\mathrm{H}_{12}$ carried by the nitrogen was the most acidic.

Table 3. NBO charge of the acidic protons of the compound 5c calculated at the $\mathrm{DFT} / \mathrm{B}_{3} \mathrm{LYP}$ level of theory.

\begin{tabular}{cc}
\hline $\mathbf{H}_{\mathrm{i}}$ & Charge \\
\hline $\mathrm{H}_{3}$ & 0.25 \\
$\mathrm{H}_{5}$ & 0.22 \\
$\mathrm{H}_{12}$ & 0.39 \\
$\mathrm{H}_{14}$ & 0.22 \\
\hline
\end{tabular}

\subsection{Antioxidant Activities}

The imbalance between reactive oxygen species (ROS) and antioxidant defence mechanisms leads to oxidative modification in cellular membrane or intracellular molecules. Phytochemicals like phenolics, commonly found in plants, could constitute strong natural antioxidants and could play an important role in health care system.

The effect of the different synthetic compounds on DPPH radical scavenging was compared to those of Trolox, using as positive control, and appreciated by the determination of the $\mathrm{IC}_{50}$ values. The results are shown in Figure 2 and listed in Table 4.

Figure 2. Scavenging effect on 1,1-diphenyl-2-picrylhydrazyl (DPPH) radical of compound 5.

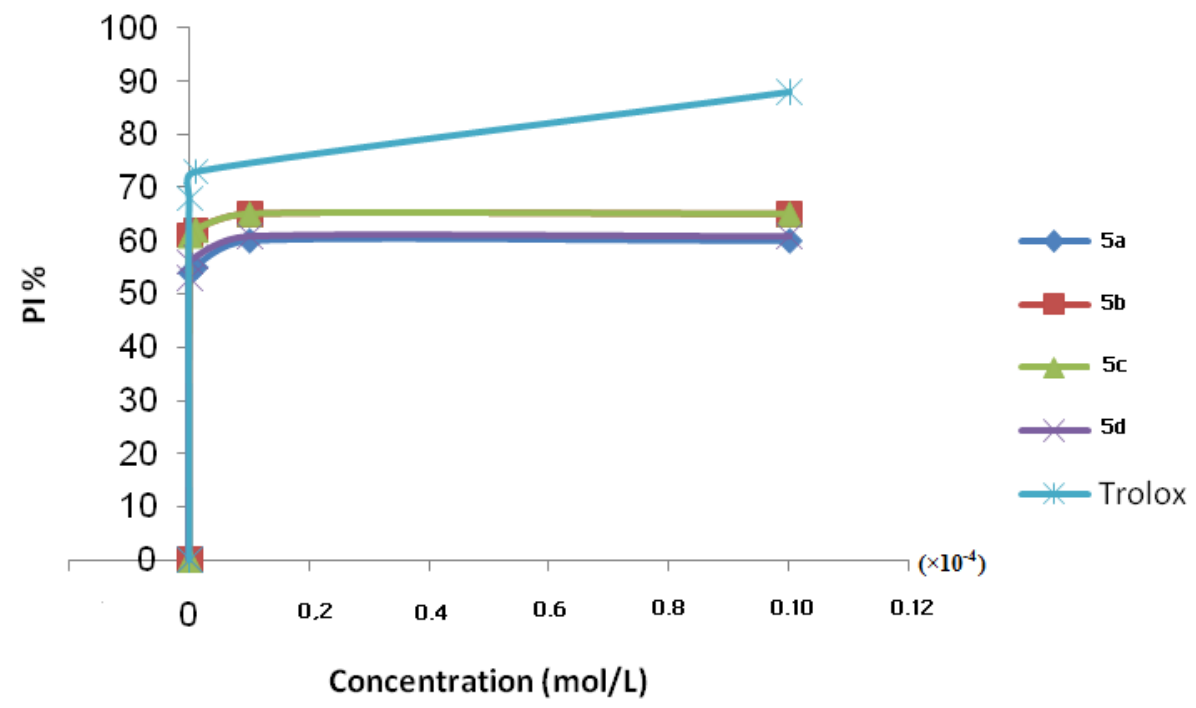

Table 4. Values of IC50 exhibited by coumarinic derivatives $\mathbf{5 a}-\mathbf{d}$.

\begin{tabular}{cc}
\hline Compounds 5 & IC $_{\mathbf{5 0}}\left(\mathbf{1 0}^{-\mathbf{9}} \mathbf{~ m o l} / \mathbf{L}\right)$ \\
\hline $\mathbf{5 a}$ & 92.60 \\
$\mathbf{5 b}$ & 82.00 \\
$\mathbf{5 c}$ & 8.62 \\
$\mathbf{5 d}$ & 9.43 \\
Trolox & 7.35 \\
\hline
\end{tabular}


As shown in Figure 2, DPPH test revealed that increase in compounds concentration resulted in increase in free radical-scavenging activity in a dose dependent manner. Based on the $\mathrm{IC}_{50}$ values, the most active compound is $\mathbf{5 c}$.

\subsection{ABTS Radical Cation Decolourization Assay}

As shown for DPPH scavenging, the obtained results indicate the higher capacity of $\mathbf{5 a}-\mathbf{d}$ to quench ABTS as compared to the synthetic antioxidant Trolox (Figure 3 and Table 5).

Figure 3. Scavenging ability on ABTS radical of compounds 5.

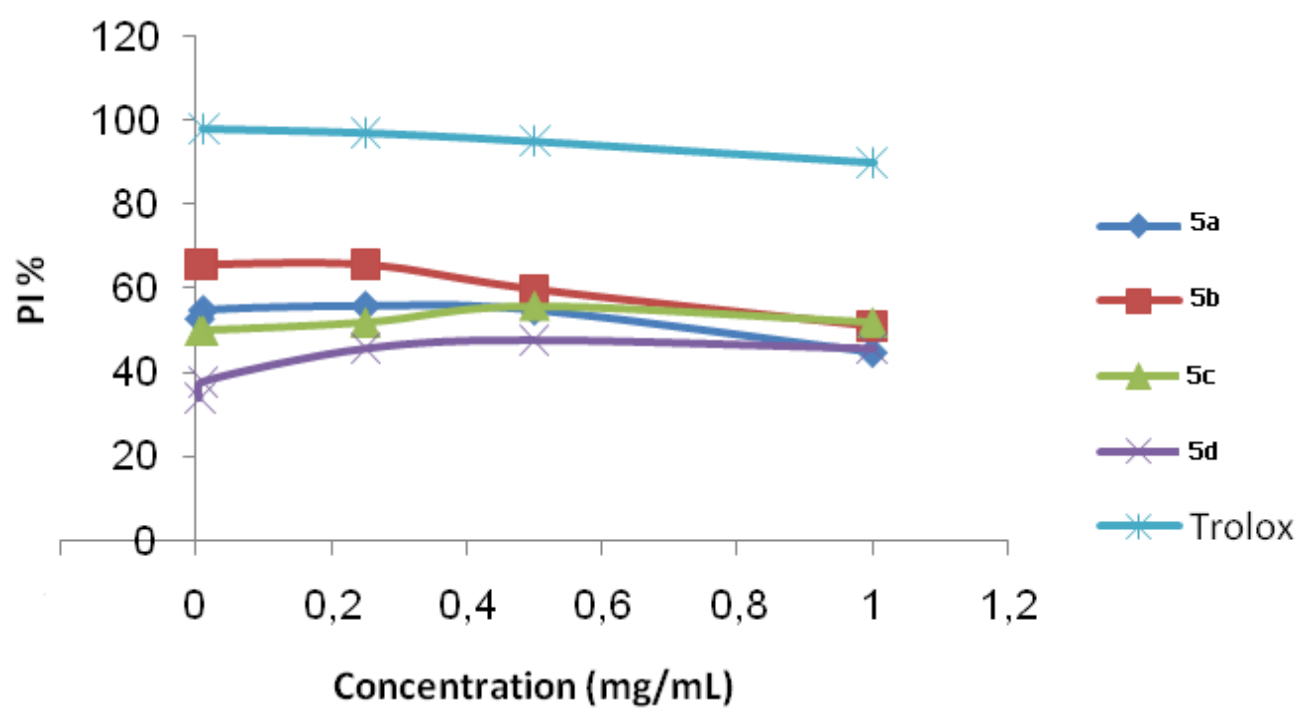

Table 5. Values of IC50 exhibited by coumarinic derivatives 5a-d.

\begin{tabular}{cc}
\hline Compounds $\mathbf{5}$ & $\mathbf{I C}_{\mathbf{5 0}}\left(\mathbf{1 0}^{\mathbf{- 9}} \mathbf{~ m o l} / \mathbf{L}\right)$ \\
\hline $\mathbf{5 a}$ & 92.60 \\
$\mathbf{5 b}$ & 82.00 \\
$\mathbf{5 c}$ & 8.62 \\
$\mathbf{5 d}$ & 9.43 \\
Trolox & 7.35 \\
\hline
\end{tabular}

\subsection{Antibacterial Activity}

The antibacterial activity of the tested compounds against a series of bacteria and fungi is shown in Table 6.

Table 6. Antibacterial activity against bacteria of 5a-d, inhibition zone expressed in $\mathrm{mm}$.

\begin{tabular}{cccccc}
\hline \multirow{2}{*}{ Organism } & \multicolumn{4}{c}{ Compounds } & Standard-drug \\
\cline { 2 - 5 } & $\mathbf{5 a}$ & $\mathbf{5 b}$ & $\mathbf{5 c}$ & $\mathbf{5 d}$ & ampicillin \\
\hline E. coli ATCC1225 & 19 & 19 & 19 & 19 & 16 \\
$\boldsymbol{P}$. vulgaris & 20 & 20 & 20 & 20 & 22 \\
B. megaterium & 17 & 17 & 17 & 17 & 23 \\
S. aureus ATCC2353 & 18 & 18 & 18 & 18 & 25 \\
\hline
\end{tabular}


All the compounds $\mathbf{5}$ displayed maximum activity against $P$. vulgaris. The compound $\mathbf{5 b}$ is highly active against E. coli. The compounds $\mathbf{5 b}$ and $\mathbf{5 d}$ also showed very good activity against B. megaterium, while compounds $\mathbf{5 a}$ and $\mathbf{5 c}$ showed good activity against $S$. aureus.

Our results also showed that the purified compounds had great potential for antibacterial activity against a panel of microorganisms. There is evidence in the literature that Gram-positive bacteria are more sensitive to plant extracts and essential oil than Gram-negative bacteria [22]. On the basis of inhibition zone diameters values $P$. vulgaris was more sensitive to the purified compounds than the other Gram positive bacteria. The observed differences in the inhibition zones within pathogenic bacteria could be probably due to cell membrane permeability or other genetic factors.

\section{Experimental}

\subsection{General}

All reagents were obtained from commercial sources. Solvents were either commercially obtained as analytical grade or freshly distilled prior to use. Analytical thin layer chromatography was carried out on precoated silica gel 60F254 plates using either UV absorption or iodine staining for visualization. Column chromatography was carried out using 100-200 mesh silica gel. ${ }^{1} \mathrm{H}-\mathrm{NMR}$ and ${ }^{13} \mathrm{C}\left\{{ }^{1} \mathrm{H}\right\}$-NMR spectra were recorded on a Bruker DRX $300 \mathrm{MHz}$ instrument (300/75 MHz); chemical shift values are reported relative to tetramethylsilane as internal standard. The IR spectra were recorded on a Thermo Mattson IR300 and Nicolet 510 PET spectrometers. Elemental microanalysis was carried out using a model 5500-Carlo Erba C.H.N.S.O elemental analyzer instrument.

General procedure for the preparation of ethyl 2-((4-methyl-2-oxo-2H-chromen-7-yl)oxy)acetate (2). A mixture of 7-hydroxy-4-methylcoumarin $(5.6 \mathrm{mmol})$, anhydrous potassium carbonate $(5.6 \mathrm{mmol})$ and ethyl bromoacetate $(5.6 \mathrm{mmol})$ in dry acetone $(10 \mathrm{~mL})$ was refluxed with continuous stirring for $12 \mathrm{~h}$. After filtration, the solution was concentrated under reduced pressure, vacuum dried and the solid product was recrystallized from ethanol. m.p. $185-186{ }^{\circ} \mathrm{C}$, yield $92 \%$; IR: $v_{\max } \mathrm{CO}$ lactone: $1,714 \mathrm{~cm}^{-1}$, NH: 3,435 $\mathrm{cm}^{-1}$, CO (ketone): $1,606 \mathrm{~cm}^{-1} ;{ }^{1} \mathrm{H}-\mathrm{NMR}: \delta 7.76\left(\mathrm{~d}, J_{5.6}=14.6 \mathrm{~Hz}, 1 \mathrm{H}, \mathrm{H}-5\right)$, $7.04\left(\mathrm{~d}, J \mathrm{H}_{5.6}=14.6 \mathrm{~Hz}, 1 \mathrm{H}, \mathrm{H}-6\right), 7.02(\mathrm{~s}, 1 \mathrm{H}, \mathrm{H}-8), 6.34$ (s, 1H, H-3), $4.92\left(\mathrm{~s}, 2 \mathrm{H},-\mathrm{OCH}_{2}\right), 4.19$ (q, $\left.J \mathrm{H}_{2}-\mathrm{H}_{3}=10.6 \mathrm{~Hz}, 2 \mathrm{H}, \mathrm{CH}_{2},-\mathrm{CH}_{2} \mathrm{CH}_{3}\right), 4.02\left(\mathrm{~s}, 2 \mathrm{H}, \mathrm{CH}_{2}\right), 1.22\left(\mathrm{t}, J \mathrm{H}_{2}-\mathrm{H}_{3}=10.6 \mathrm{~Hz}, 3 \mathrm{H}, \mathrm{CH}_{3}\right.$, - $\left.\mathrm{CH}_{2} \mathrm{CH}_{3}\right) ;{ }^{13} \mathrm{C}\left\{{ }^{1} \mathrm{H}\right\}$ - NMR: $\delta 14.2\left(\mathrm{CH}_{2} \mathrm{CH}_{3}\right), 34.8\left(\mathrm{CH}_{2} \mathrm{CO}\right), 61.3\left(\mathrm{CH}_{2} \mathrm{CH}_{3}\right), 65.5\left(\mathrm{COCH}_{2} \mathrm{O}\right), 109.6$ (C-8), 112.8 (C-6), 113.8 (C-3), 114.8 (C-10), 128.3 (C-5), 151.2 (C-9), 155.2 (C-4), 160.3 (C-7), 160.9 (C-2), 168.9 (CO-O), 169.3 (C-CO-C). Found, \%: C, 64.12; H, 5.2; O, 30.40. $\mathrm{C}_{14} \mathrm{H}_{14} \mathrm{O}_{5}$. Calculated, \%: C, 64.12; H, 5.38; O, 30.50 .

2-(4-Methyl-2-oxo-2H-chromen-7-yloxy)acetohydrazide (3). To a solution of ethanol (15 mL) and hydrazine hydrate $(10 \mathrm{mmol})$, ethyl 2-(4-methyl-2-oxo- $2 H$-chromen-7-yloxy) acetate $(2,10 \mathrm{mmol})$ was added, and the mixture was refluxed for $4 \mathrm{~h}$. The product precipitated and was collected by suction filtration, washed with methanol and recrystallized from dil. Acetic acid. m.p. $=300{ }^{\circ} \mathrm{C}$, yield 70\%; IR: $\nu_{\max }$ CO lactone: $1,681 \mathrm{~cm}^{-1}, \mathrm{CONH}$ (amide): $1,612 \mathrm{~cm}^{-1}, \mathrm{CN}: 1,271 \mathrm{~cm}^{-1}$; ${ }^{1} \mathrm{H}-\mathrm{NMR}: \delta 9.41(\mathrm{~s}, 1 \mathrm{H}$, $\mathrm{NH}), 7.76\left(\mathrm{~d}, \mathrm{H}_{5.6}=14.6 \mathrm{~Hz}, 1 \mathrm{H}, \mathrm{H}-5\right), 7.04$ (d, JH $\left.\mathrm{H}_{5.6}=14.6 \mathrm{~Hz}, 1 \mathrm{H}, \mathrm{H}-6\right), 7.02$ (s, 1H, H-8), 6.34 (s, $1 \mathrm{H}, \mathrm{H}-3), 4.94\left(\mathrm{~s}, 2 \mathrm{H},-\mathrm{OCH}_{2}\right), 4.34\left(\mathrm{~s}, 2 \mathrm{H}, \mathrm{NH}_{2}\right) .{ }^{13} \mathrm{C}\left\{{ }^{1} \mathrm{H}\right\}-\mathrm{NMR}: \delta 45.8\left(\mathrm{CH}_{2}\right), 68.9\left(\mathrm{CH}_{2} \mathrm{O}-\right), 108.0$ 
(C-8), 111.8 (C-6), 112.9 (C-3), 114.1 (C-10), 128.3 (C-5), 152.2 (C-9), 155.2 (C-4), 160.4 (C-7), $160.9(\mathrm{C}-2), 166.8\left(\mathrm{COCH}_{2} \mathrm{O}\right), 169.6\left(\underline{\mathrm{COCH}}_{2}\right)$. Found, \%: C, 58.1; H, 4.82; N, 11.1; O, 25.70 $\mathrm{C}_{12} \mathrm{H}_{12} \mathrm{O}_{4} \mathrm{~N}_{2}$. Calculated, \%: C, 58.06; H, 4.87; N, 11.29; O, 25.78.

General procedure for the preparation of $N^{\prime}$-benzylidene-2-(4-methyl-2-oxo-2H-chromen-7-yloxy) acetohydrazide (4). A mixture of 2-(4-methyl-2-oxo- $2 H$-chromen-7-yloxy)acetohydrazide (3, $3.06 \mathrm{~g}$, $0.01 \mathrm{~mol}$ ) and appropriate aromatic aldehyde (Ar/a-d, $0.01 \mathrm{~mol})$ was refluxed in absolute ethanol (30 $\mathrm{mL}$ ) in the presence of a catalytic amount of glacial acetic for $4 \mathrm{~h}$. The reaction mixture was cooled; the solid separated was filtered and recrystallized from methanol to give compounds $\mathbf{4 a}-\mathbf{d}$.

$N^{\prime}$-Benzylidene-2-((4-methyl-2-oxo-2H-chromen-7-yl)oxy)acetohydrazide (4a). m.p. 268-269 ${ }^{\circ} \mathrm{C}$; yield 74\%; IR: $v_{\max } \mathrm{CO}$ lactone: $1681 \mathrm{~cm}^{-1}, \mathrm{CONH}$ (amide): $1610 \mathrm{~cm}^{-1}, \mathrm{C}=\mathrm{N}: 1258 \mathrm{~cm}^{-1} ;{ }^{1} \mathrm{H}-\mathrm{NMR}: \delta 8.30$ $(\mathrm{s}, 1 \mathrm{H}, \mathrm{HC}=\mathrm{N}-), 8.02(\mathrm{~s}, 1 \mathrm{H}, \mathrm{NH}), 7.76\left(\mathrm{~d}, \mathrm{H}_{5.6}=15.6 \mathrm{~Hz}, 1 \mathrm{H}, \mathrm{H}-5\right), 7.72-7.31(\mathrm{~m}, 10 \mathrm{H}$, arom. $), 7.04$ $\left(\mathrm{d}, \mathrm{H}_{5.6}=15.6 \mathrm{~Hz}, 1 \mathrm{H}, \mathrm{H}-6\right), 7.02(\mathrm{~s}, 1 \mathrm{H}, \mathrm{H}-8), 6.34(\mathrm{~s}, 1 \mathrm{H}, \mathrm{H}-3), 4.83\left(\mathrm{~s}, 2 \mathrm{H},-\mathrm{OCH}_{2}\right),{ }^{13} \mathrm{C}\left\{{ }^{1} \mathrm{H}\right\}-$ NMR: $\delta 45.7\left(\mathrm{CH}_{2}\right), 69.2\left(\mathrm{CH}_{2} \mathrm{O}-\right), 108.1(\mathrm{C}-8), 111.6$ (C-6), 112.5 (C-3), 114.4 (C-10), $127.9(\mathrm{C}-5)$, 128.4 (C-3,5, Ar-), 129.0 (C-2,6, Ar-), 131.4 (C-4, Ar-), 133.9 (C-1, Ar-), 143.6 (N=CH-), 151.8 (C-9), 155.2 (C-4), 160.4 (C-7), 160.9 (C-2), 166.9 ( $\mathrm{COCH}_{2} \mathrm{O}$ ), 170.0 (CONH-). Found, \%: C, 67.75; H, 4.8; $\mathrm{N}, 8.2 ; \mathrm{O}, 19.1 . \mathrm{C}_{19} \mathrm{H}_{16} \mathrm{O}_{4} \mathrm{~N}_{2}$. Calculated, \%: C, 67.85; H, 4.79; N, 8.33; O, 19.03.

N'-(3-Methoxybenzylidene)-2-((4-methyl-2-oxo-2H-chromen-7-yl)oxy)acetohydrazide (4b). m.p. 225-226 ${ }^{\circ} \mathrm{C}$, yield (76\%); IR: $v_{\max } \mathrm{CO}$ lactone:1,681 $\mathrm{cm}^{-1}, \mathrm{CONH}$ (amide): 1,614 $\mathrm{cm}^{-1}, \mathrm{C}=\mathrm{N}$ : 1,255 $\mathrm{cm}^{-1}$; ${ }^{1} \mathrm{H}-\mathrm{NMR}: \delta 8.73(\mathrm{~s}, 1 \mathrm{H},-\mathrm{HC}=\mathrm{N}-), 8.45(\mathrm{~s}, 1 \mathrm{H}, \mathrm{NH}), 7.72\left(\mathrm{~d}, \mathrm{JH}_{5.6}=15.5 \mathrm{~Hz}, 1 \mathrm{H}, \mathrm{H}-5\right)$, 7.60-7.30 (m, 8H, arom.), 3.65 (s, 3H, $\mathrm{OCH}_{3}$ ), 7.04 (d, JH $\left.\mathrm{J}_{5.6}=15.4 \mathrm{~Hz}, 1 \mathrm{H}, \mathrm{H}-6\right), 7.02$ (s, 1H, H-8), $6.34(\mathrm{~s}, 1 \mathrm{H}, \mathrm{H}-3), 4.84\left(\mathrm{~s}, 2 \mathrm{H},-\mathrm{OCH}_{2}\right),{ }^{13} \mathrm{C}\left\{{ }^{1} \mathrm{H}\right\}$ NMR: $\delta 45.6\left(\mathrm{CH}_{2}\right), 68.9\left(\mathrm{CH}_{2} \mathrm{O}-\right), 107.8(\mathrm{C}-8)$, 111.5(C-6), 112.7 (C-3), 113.9 (C-10), 127.8 (C-5), 129.4 (C-3, Ar-), 130.6 (C-6, Ar-), 132.7 (C-4, Ar-),133.8 (C-1, Ar-), 134.3 (C-2, Ar-), 143.5 (N=CH-), 151.7 (C-9), 155.0 (C-4), 160.3 (C-7), 160.9 (C-2), $166.4\left(\mathrm{COCH}_{2} \mathrm{O}\right), 169.9$ (CONH-). Found, \%: C, 65.5; H, 4.80; N, 7.6; O, 21.8. $\mathrm{C}_{20} \mathrm{H}_{18} \mathrm{O}_{5} \mathrm{~N}_{2}$. Calculated, \%: C, 65.57; H, 4.95; N, 7.65; O, 21.84.

$N^{\prime}$-(2.3.4-Trimethoxybenzylidene)-2-((4-methyl-2-oxo-2H-chromen-7-yl)oxy)acetohydrazide (4c). m.p. 259-261 ${ }^{\circ} \mathrm{C}$, yield 72\%; IR: $v_{\max } \mathrm{CO}$ lactone: $1,681 \mathrm{~cm}^{-1}, \mathrm{CONH}$ (amide): $1618 \mathrm{~cm}^{-1}, \mathrm{C}=\mathrm{N}: 1,281 \mathrm{~cm}^{-1}$. ${ }^{1} \mathrm{H}-\mathrm{NMR}: \delta 8.31(\mathrm{~s}, 1 \mathrm{H},-\mathrm{HC}=\mathrm{N}-), 7.76\left(\mathrm{~d}, \mathrm{H}_{5.6}=15.5 \mathrm{~Hz}, 1 \mathrm{H}, \mathrm{H}-5\right), 7.67-7.30(\mathrm{~m}, 8 \mathrm{H}$, arom.), 7.04 $\left(\mathrm{d}, J \mathrm{H}_{5.6}=15.6 \mathrm{~Hz}, 1 \mathrm{H}, \mathrm{H}-6\right), 7.02(\mathrm{~s}, 1 \mathrm{H}, \mathrm{H}-8), 6.34$ (s, 1H, H-3), 3.78 (s, 9H, 2,3,4 OCH OC, $_{4} .84$ $\left(\mathrm{s}, 2 \mathrm{H},-\mathrm{OCH}_{2}\right),{ }^{13} \mathrm{C}\left\{{ }^{1} \mathrm{H}\right\}-\mathrm{NMR}: \delta 45.5\left(\mathrm{CH}_{2}\right), 69.1\left(\mathrm{CH}_{2} \mathrm{O}-\right), 107.9(\mathrm{C}-8), 111.4(\mathrm{C}-6), 112.5(\mathrm{C}-3)$, 113.4 (C-10), 127.3 (C-6, Ar-), 127.9 (C-5), 129.3 (C-2, Ar-), 130.3 (C-5, Ar-), 131.2 (C- 4, Ar-), 135.2 (C-1, Ar-), 134.4 (C-3, Ar-), 143.4 (N=CH-), 151.6 (C-9), 155.2 (C-4), 160.6 (C-7), 160.9 (C-2), $166.7\left(\mathrm{COCH}_{2} \mathrm{O}\right), 169.8$ (CONH-). Found, \%: C, 61.9; H, 5.1; N, 6.5; O, 26.3. $\mathrm{C}_{22} \mathrm{H}_{22} \mathrm{O}_{7} \mathrm{~N}_{2}$. Calculated, \%: C, 61.97; H, 5.20; N, 6.57; O, 26.26.

$N^{\prime}$-(4-Bromobenzylidene)-2-((4-methyl-2-oxo-2H-chromen-7-yl)oxy)acetohydrazide (4d). m.p. 273-275 ${ }^{\circ} \mathrm{C}$, yield 52\%; IR: $v_{\max } \mathrm{CO}$ lactone: $1,681 \mathrm{~cm}^{-1}, \mathrm{CONH}$ (amide): $1,612 \mathrm{~cm}^{-1},{ }^{1} \mathrm{H}-\mathrm{NMR}: 8.30$ $(\mathrm{s}, 1 \mathrm{H},-\mathrm{HC}=\mathrm{N}-), 8.23(\mathrm{~s}, 1 \mathrm{H}, \mathrm{NH}), 7.76\left(\mathrm{~d}, \mathrm{H}_{5.6}=15.5 \mathrm{~Hz}, 1 \mathrm{H}, \mathrm{H}-5\right), 7.61-7.30(\mathrm{~m}, 6 \mathrm{H}$, arom.), 7.04 $\left(\mathrm{d}, J \mathrm{H}_{5.6}=15.5 \mathrm{~Hz}, 1 \mathrm{H}, \mathrm{H}-6\right), 7.02(\mathrm{~s}, 1 \mathrm{H}, \mathrm{H}-8), 6.34(\mathrm{~s}, 1 \mathrm{H}, \mathrm{H}-3), 4.82\left(\mathrm{~s}, 2 \mathrm{H},-\mathrm{OCH}_{2}\right),{ }^{13} \mathrm{C}\left\{{ }^{1} \mathrm{H}\right\}-$ 
NMR: $\delta 45.6\left(\mathrm{CH}_{2}\right), 69.2\left(\mathrm{CH}_{2} \mathrm{O}-\right), 103.8$ (C-3, Ar-), 107.6 (C-8), 108.7 (C-5, Ar-),111.3 (C-6), 112.7 (C-3), 113.4 (C-10), 127.2 (C-6, Ar-), 127.9 (C-5), 135.2 (C-1, Ar-), 143.0 (N=CH-), 151.2 (C-9), 155.1 (C-4), 160.5 (C-7), 160.9 (C-2), 162.4 (C-2, Ar-), 162.6 (C-4, Ar-), $166.5\left(\mathrm{COCH}_{2} \mathrm{O}\right), 169.4$ (CONH-). Found, \%: C, 54.8; H, 3.6; Br, 19.1; N, 6.7; O, 15.4. $\mathrm{C}_{19} \mathrm{H}_{15} \mathrm{O}_{4} \mathrm{~N}_{2}$. Calculated, \%: C, 54.96; H, 3.64; Br, 19.24; N, 6.75; O, 15.41 .

General procedure for the preparation of 2-(4-methyl-2-oxo-2H-chromen-7-yloxy)-N-(4-oxo-2 arylthiazolidin-3-yl) acetamide (5). A mixture of $N^{\prime}$-benzylidene-2-(4-methyl-2-oxo-2H-chromen-7yloxy) acetohydrazide (4, $0.01 \mathrm{~mol})$ and mercaptoacetic acid (1.82 g, $0.02 \mathrm{~mol})$ in 1,4-dioxane $(30 \mathrm{~mL})$ containing a pinch of anhydrous $\mathrm{ZnCl}_{2}$ was refluxed for 6-8 h. The reaction mixture was cooled and poured onto crushed ice. The solid thus obtained was filtered, washed with water and recrystallized from DMF yielding $\mathbf{5 a}-\mathbf{d}$.

2-(4-Methyl-2-oxo-2H-chromen-7-yloxy)-N-(4-oxo-2-phenylthiazolidin-3-yl)acetamide (5a). m.p. 202-204 ${ }^{\circ} \mathrm{C}$, yield 40\%; IR: $v_{\max } \mathrm{CO}$ lactone: $1,681 \mathrm{~cm}^{-1}, \mathrm{CONH}$ (amide): $1,615 \mathrm{~cm}^{-1}$; ${ }^{1} \mathrm{H}-\mathrm{NMR}: 2.15$ (s, 3H), $8.12(\mathrm{~s}, 1 \mathrm{H},-\mathrm{NH}), 7.76\left(\mathrm{~d}, \mathrm{H}_{5.6}=10.6 \mathrm{~Hz}, 1 \mathrm{H}, \mathrm{H}-5\right), 7.71-7.23$ (m, 10H, arom.), 7.04 (d, $\left.J_{5.6}=10.6 \mathrm{~Hz}, 1 \mathrm{H}, \mathrm{H}-6\right), 7.02(\mathrm{~s}, 1 \mathrm{H}, \mathrm{H}-8), 6.34(\mathrm{~s}, 1 \mathrm{H}, \mathrm{H}-3), 5.92(\mathrm{~s}, 1 \mathrm{H},-\mathrm{SCHN}-), 4.83$ (s, $\left.2 \mathrm{H},-\mathrm{OCH}_{2}\right), 3.38\left(\mathrm{~s}, 2 \mathrm{H}, \mathrm{COCH}_{2} \mathrm{~S}-\right) ;{ }^{13} \mathrm{C}\left\{{ }^{1} \mathrm{H}\right\}-\mathrm{NMR}: \delta 35.8\left(\mathrm{COCH}_{2} \mathrm{~S}\right), 45.5\left(\mathrm{CH}_{2}\right), 57.4(\mathrm{NCHS})$, $69.1\left(\mathrm{CH}_{2} \mathrm{O}-\right)$ 107.6 (C-8), 111.0 (C-6), 112.5 (C-3), 113.4 (C-10), 127.2 (C-4, Ar-), 127.8 (C-5), 128.7 (C-3,5, Ar-),128.8 (C-2,6 Ar-), 139.2 (C-1, Ar-), 151.2 (C-9), 155.0 (C-4), 160.3 (C-7), 160.9 (C-2), $166.4\left(\mathrm{COCH}_{2} \mathrm{O}\right), 168.8\left(\mathrm{SCH}_{2} \mathrm{CO}-\mathrm{N}\right), 173.3(\mathrm{CONH}-)$. Found, \%: C, 61.3; H, 4.4; N, 6.8; O, 19.5; S, 7.7. $\mathrm{C}_{21} \mathrm{H}_{18} \mathrm{O}_{5} \mathrm{~N}_{2}$ S. Calculated, \%: C, 61.45; H, 4.42; N, 6.83; O, 19.49; S, 7.81.

N-(2-(3-Methoxyphenyl)-4-oxothiazolidin-3-yl)-2-(4-methyl-2-oxo-2H-chromen-7-yloxy)acetamide $(5 \mathbf{b})$. m.p. $184{ }^{\circ} \mathrm{C}$, yield 76\%; IR: $v_{\max } \mathrm{CO}$ lactone: $1,681 \mathrm{~cm}^{-1}$, CONH (amide): $1,614 \mathrm{~cm}^{-1}$; ${ }^{1} \mathrm{H}-\mathrm{NMR}: 8.62$ (s, 1H, NH-), 2.20 (s, 3H), 7.76 (d, JH $\left.\mathrm{H}_{5.6}=10.5 \mathrm{~Hz}, 1 \mathrm{H}, \mathrm{H}-5\right), 7.60-7.30$ (m, 8H, arom.), 3.67 (s, 3H, $\left.\mathrm{OCH}_{3}\right), 7.04\left(\mathrm{~d}, \mathrm{H}_{5.6}=10.5 \mathrm{~Hz}, 1 \mathrm{H}, \mathrm{H}-6\right), 7.02$ (s, 1H, H-8), 6.34 (s, 1H, H-3), 5.92 (s, 1H, NCHS), $4.84\left(\mathrm{~s}, 2 \mathrm{H},-\mathrm{OCH}_{2}\right), 3.38\left(\mathrm{~s}, 2 \mathrm{H}, \mathrm{COCH}_{2} \mathrm{~S}\right) ;{ }^{13} \mathrm{C}\left\{{ }^{1} \mathrm{H}\right\}-\mathrm{NMR}: \delta 35.7\left(\mathrm{COCH}_{2} \mathrm{~S}\right), 45.5\left(\mathrm{CH}_{2}\right), 57.4$ (NCHS), 69.10 ( $\left.\mathrm{CH}_{2} \mathrm{O}-\right)$, 107.6 (C-8), 111.0 (C-6), 112.5 (C-3), 113.4 (C-10), 127.0 (C-5), 129.0 (C-3, Ar-), 130.6 (C-6, Ar-), 132.5 (C-4, Ar-), 133.4 (C-1, Ar-), 134.0 (C-2, Ar-), 143.0 (N=CH-), 151.2 (C-9), 155.0 (C-4), 160.3 (C-7), 160.9 (C-2), 166.4 ( $\left.\mathrm{COCH}_{2} \mathrm{O}\right), 173.0$ (CONH-). Found, \%: C, 59.9; H, 4.5; N, 6.4; O, 21.8; S, 7.2. $\mathrm{C}_{22} \mathrm{H}_{20} \mathrm{O}_{6} \mathrm{~N}_{2} \mathrm{~S}$. Calculated, \%: C, 59.99; H, 4.58; N, 6.36; O, 21.79; S, 7.28.

2-(4-Methyl-2-oxo-2H-chromen-7-yloxy)-N-(4-oxo-2-(2,3,4-trimethoxyphenyl)thiazolidin-3-yl)acetamide (5c). m.p. 239-241 ${ }^{\circ} \mathrm{C}$, yield 52\%; IR: $v_{\max } 1,712\left(\mathrm{C}=\mathrm{O}\right.$, lactone), 1,624 (C=O, amide) $\mathrm{cm}^{-1}$; ${ }^{1} \mathrm{H}-\mathrm{NMR}$ : $2.12(\mathrm{~s}, 3 \mathrm{H}), 8.30(\mathrm{~s}, 1 \mathrm{H}, \mathrm{NH}-), 7.76\left(\mathrm{~d}, J_{5.6}=10.4 \mathrm{~Hz}, 1 \mathrm{H}, \mathrm{H}-5\right), 7.61-7.30$ (m, 6H, arom.), 7.04 $\left(\mathrm{d}, J \mathrm{H}_{5.6}=10.4 \mathrm{~Hz}, 1 \mathrm{H}, \mathrm{H}-6\right), 7.02(\mathrm{~s}, 1 \mathrm{H}, \mathrm{H}-8), 6.34(\mathrm{~s}, 1 \mathrm{H}, \mathrm{H}-3), 5.92$ (s, 1H, NCHS), 4.82 $\left(\mathrm{s}, 2 \mathrm{H},-\mathrm{OCH}_{2}\right), 3.75\left(\mathrm{~s}, 9 \mathrm{H}, 2,3,4 \mathrm{OCH}_{3}\right), 4.28\left(\mathrm{~s}, 2 \mathrm{H}, \mathrm{CH}_{2}\right), 3.38\left(\mathrm{~s}, 2 \mathrm{H}, \mathrm{COCH}_{2} \mathrm{~S}\right) ;{ }^{13} \mathrm{C}\left\{{ }^{1} \mathrm{H}\right\}-\mathrm{NMR}: \delta$ $35.7\left(\mathrm{COCH}_{2} \mathrm{~S}\right), 45.5\left(\mathrm{CH}_{2}\right), 47.4$ (NCHS), $69.10\left(\mathrm{CH}_{2} \mathrm{O}-\right), 103.7$ (C-3, Ar-), $107.6(\mathrm{C}-8), 108.4(\mathrm{C}-5$, Ar-), 110.1 (C-1, Ar-), 111.0 (C-6), 112.5 (C-3), 113.4 (C-10), 127.8 (C-5),131.3 (C-6, Ar-), 151.2 (C-9), 157.2 (C-2, Ar-), 158.2 (C-4), 160.3 (C-7), 160.9 (C-2), 166.4 (CONH), 168.8 ( $\left.\mathrm{NCOCH}_{2}\right), 173.3$ $\left(\mathrm{CH}_{2} \mathrm{CONH}\right)$. Found, \%: C, 57.4; H, 4.75; N, 5.7; O, 25.6; S, 6.5. $\mathrm{C}_{24} \mathrm{H}_{24} \mathrm{O}_{8} \mathrm{~N}_{2} \mathrm{~S}$. Calculated, \%: C, $57.59 ; \mathrm{H}, 4.83 ; \mathrm{N}, 5.60 ; \mathrm{O}, 25.57 ; \mathrm{S}, 6.41$. 
N-(2-(4-Bromophenyl)-4-oxothiazolidin-3-yl)-2-(4-methyl-2-oxo-2H-chromen-7-yloxy)acetamide (5d). m.p. $240-241{ }^{\circ} \mathrm{C}$, yield 72\%; IR: $v_{\max } 1,727\left(\mathrm{CO}\right.$, lactone), 1,616 (C=O, amide) $\mathrm{cm}^{-1}$; ${ }^{1} \mathrm{H}-\mathrm{NMR}: 2.14$ (s, 3H), 8.22 (s, 1H, NH-), $7.76\left(\mathrm{~d}, \mathrm{H}_{5.6}=10.5 \mathrm{~Hz}, 1 \mathrm{H}, \mathrm{H}-5\right), 7.67-7.30$ (m, 8H, arom.), 7.04 (d, $\left.J_{5.6}=10.5 \mathrm{~Hz}, 1 \mathrm{H}, \mathrm{H}-6\right), 7.02(\mathrm{~s}, 1 \mathrm{H}, \mathrm{H}-8), 6.34$ (s, 1H, H-3), 5.92 (s, 1H, NCHS), 4.84 (s, 2H, $\left.-\mathrm{OCH}_{2}\right), 3.38\left(\mathrm{~s}, 2 \mathrm{H}, \mathrm{COCH}_{2} \mathrm{~S}\right) ;{ }^{13} \mathrm{C}\left\{{ }^{1} \mathrm{H}\right\}-\mathrm{NMR}: \delta 35.7\left(\mathrm{COCH}_{2} \mathrm{~S}\right), 45.5\left(\mathrm{CH}_{2}\right), 57.4(\mathrm{NCHS}), 69.10$ $\left(\mathrm{CH}_{2} \mathrm{O}-\right)$, 107.6 (C-8), 111.0 (C-6), 112.5 (C-3), 113.4 (C-10), 127.3 (C-6, Ar-), 127.8 (C-5), 129.3 (C-2, Ar-), 130.3 (C-5, Ar-), 131.2 (C-4, Ar-), 135.2 (C-1, Ar-), 134.4 (C-3, Ar-), 143.0 (N=CH-), 151.2 (C-9), 155.0 (C-4), 160.3 (C-7), 160.9 (C-2), 173.0 ( $\left.\mathrm{COCH}_{2} \mathrm{O}\right), 173.0$ (CONH-). Found, \%: C, 51.6; H, 3.4; Br, 16.4; N, 5.75; O, 16.4; S, 6.7. $\mathrm{C}_{21} \mathrm{H}_{17} \mathrm{O}_{5} \mathrm{~N}_{2} \mathrm{BrS}$. Calculated, \%: C, 51.54; H, 3.50; $\mathrm{Br}$, $16.33 ; \mathrm{N}, 5.72 ; \mathrm{O}, 16.35 ; \mathrm{S}, 6.55$.

\subsection{DPPH Test}

The DPPH test aims to measure the capacity of the compounds for scavenging the stable free radical 2,2-diphenyl-1-picrylhydrazyl (DPPH) by donation of hydrogen atom or an electron[23] If the compounds have the capacity to scavenge the DPPH free radical, the initial blue/purple solution will change to a yellow colour due to the formation of diphenylpicrylhydrazine. Scavenging capacity was read spectrophotometrically by monitoring the decrease of the absorbance at $517 \mathrm{~nm}$. Lower absorbance of the reaction mixture indicates higher free radical scavenging activity.

The percent DPPH scavenging effect was calculated using the following equation:

$$
\text { DPPH scavenging effect }(\%)=\left(\mathrm{A}_{\text {control }}-\mathrm{A}_{\text {sample }} / \mathrm{A}_{\text {control }}\right) \times 100
$$

where $\mathrm{A}_{\text {control }}$ is the absorbance of the control reaction were the sample is replaced by $500 \mu \mathrm{L}$ ethanol. Tests were carried out in triplicate.

\subsection{ABTS Radical Cation Decolourization Assay}

The potential of $\mathrm{N}$-(2-aryl-4-oxo-thiazolidin-3-yl)-2-(4-(2-aryl-4-oxo-thiazolidin-3-ylcarbamoyl)methyl)-2-oxo-2H-chromen-7-yloxy)-acetamides 5a-d to scavenge free radicals was also assessed by checking their ability to quench ABTS depicted by the concentration-dependent decolourization of ABTS : ABTS radical-scavenging activity of 5a-d was determined according to Güven et al. [24]. The ABTS cation radical was produced by the reaction between $5 \mathrm{~mL}$ of $14 \mathrm{mM} \mathrm{ABTS}$ solution and $5 \mathrm{~mL}$ of $4.9 \mathrm{mM}$ potassium persulfate $\left(\mathrm{K}_{2} \mathrm{~S}_{2} \mathrm{O}_{8}\right)$ solution, stored in the dark at room temperature for $16 \mathrm{~h}$. Before use, this solution was diluted with ethanol to get an absorbance of $0.700 \pm 0.020$ at $734 \mathrm{~nm}$. In a final volume of $1 \mathrm{~mL}$, the reaction mixture comprised $950 \mu \mathrm{L}$ of ABTS solution and $50 \mu \mathrm{L}$ of $\mathbf{5 a}-\mathbf{d}$ at various concentrations. The reaction mixture was homogenized and its absorbance was recorded at $734 \mathrm{~nm}$. Ethanol blanks were run in each assay, and all measurements were done after at least 6 min. Similarly, the reaction mixture of standard group was obtained by mixing $950 \mu \mathrm{L}$ of ABTS solution and $50 \mu \mathrm{L}$ of Trolox. As for the antiradical activity, ABTS scavenging ability was expressed as $\mathrm{IC}_{50}(\mu \mathrm{g} / \mathrm{mL})$. The inhibition percentage of ABTS radical was calculated using the following formula:

ABTS scavenging effect $\%=\left\{\left[\left(\mathrm{A}_{0}-\mathrm{A}_{1}\right)\right] / \mathrm{A}_{0}\right\} \times 100 \%$ 
where $\mathrm{A}_{0}$ is the absorbance of the control at $30 \mathrm{~min}$, and $\mathrm{A} 1$ is the absorbance of the sample at $30 \mathrm{~min}$. All samples were analyzed in triplicate.

\subsection{Antibacterial Activity}

Antimicrobial activity of the purified compounds was evaluated by means of agar-well diffusion assay according to Güven et al. [25] with some modifications. The nutrient agar broth prepared by the usual method, was inoculated aseptically with $0.5 \mathrm{~mL}$ of $24 \mathrm{~h}$ old subculture of $S$. aureus ATCC 2353, B. megaterium, P. vulgaris, and E. coli ATCC1225 in separate conical flasks at $40-50{ }^{\circ} \mathrm{C}$ and mixed well by gentle shaking. About $25 \mathrm{~mL}$ of the contents of the flask were poured and evenly spread in petridish (90 $\mathrm{mm}$ in diameter) and allowed to set for two $\mathrm{h}$. The cups (10 $\mathrm{mm}$ in diameter) were formed by the help of borer in agar medium and filled with $0.04 \mathrm{~mL}(40 \mu \mathrm{g} / \mathrm{mL})$ solution of sample in DMF. The plates were incubated at $37^{\circ} \mathrm{C}$ for $24 \mathrm{~h}$ and the control was also maintained with $0.04 \mathrm{~mL}$ of DMF in similar manner and the zones of inhibition of the bacterial growth were measured in millimetre

\section{Conclusions}

A series of new coumarin-based thiazolidinone compounds were successfully synthesized, characterized and tested for their antibacterial and antioxidant activities. Most of the synthesized compounds are more active against $E$. coli and $S$. aureus than standard references. Some of the compounds were found equipotent to ampicillin, but less active than other used standard drugs

\section{Acknowledgements}

This work was carried out with financial aid of the Qassim University in KSA through project No. 1303.

\section{References}

1. Geronikaki, A.; Hadjiparlon-Litina, D.; Chatzioponlos, C.; Soloupis, G. Synthesis and biological evolution of new 4, 5-disubstituted-thiazoylamides derivatives of 4-hydroxy-Piperidine or 4- $\mathrm{N}$ methyl-Piperadine. Molecules 2003, 8, 472-479.

2. Sup, R.C.; Sup, R.Y.; Bang, C.W. Synthesis and antiinflammatory activity of [2-(benzothiazol-2ylimino)-4-oxo-3-pheylthiazolidin-5-yl]-acetic acid derivatives. J. Korean Chem. Soc. 1995, 93, 237-240.

3. Sonwane, S.K.; Srivastava, S.D. Synthesis and biological significance of 2-amino-4-phenyl-1, 3-thiazole derivatives. Proc. Natl. Acad. Sci. India 2008, 78, 129-136.

4. Capan, G.; Ulusoy, N.; Ergenc, N.; Kiraz, M. New 6-phenylimidazo[2,1-b]thiazole derivatives: Synthesis and antifungal activity. Monatsh. Chem. 1999, 130, 1399-1407.

5. Vigorita, M.; Ottana, R.; Monforte, F.; Maccari, R.; Trovato, A.; Monforte, M.T.; Ftaviano, M. Synthesis and antiinflammatory, analgesic activity of 3,3'-(1,2-Ethanediyl)-bis[2-aryl-4thiazolidinone] chiral compounds. Part 10. Bioorg. Med. Chem. Lett. 2001, 11, 2791-2794. 
6. Kavitha, C.; Basappa, S.; Nanjunda, S.; Mantelingu, K.; Doreswamy, S.; Sridhar, M.A.; Sprasad, J.; Rangappa, K. Synthesis of new bioactive venlafaxine analogs: Novel thiazolidin-4-ones as antimicrobials. Bioorg. Med. Chem. 2006, 14, 2290-2299.

7. Ottana, R.; Maccari, R.; Barreca, M.; Rotondo, G.; Rossi, A.; Chiricosta, A.; Paola, G.; Sautebin, R.; Cuzzocrea, L.; Vigorita, S. Arylidene-2-imino-4-thiazolidinones: Design and synthesis of novel anti-inflammatory agents. Bioorg. Med. Chem. 2005, 13, 4243-4252.

8. Kucukguzel, G.; Kocatepe, A.; Clercq, E.; Sahin, F.; Gulluce, M. Synthesis and biological activity of 4-thiazolidinones, thiosemicarbazides derived from diflunisal hydrazide. Eur. J. Med. Chem. 2006, 41, 353-359.

9. Al-Amiery, A.A.; Musa, A.Y.; Kadhum, A.H.; Mohamad, A. The use of umbelliferone in the synthesis of new heterocyclic compounds. Molecules 2011, 16, 6833-6843.

10. Al-Amiery, A. Kadhum, A.; Mohamad, A. Antifungal activities of new coumarins. Molecules 2012, 17, 5713-5723.

11. Al-Amiery, A.A.; Musa, A.Y.; Kadhum, A.H.; Mohamad, A.B. The antioxidant activity of new coumarin derivatives. Int. J. Mol. Sci. 2011, 12, 5757-5761.

12. Ocal, N.; Yolacan, C.; Kaban, S.; Leonor, Y.; Vargas, M.; Kouznetsov, V.J. Transformations of schiff bases derived from quinoline-8-carbaldehyde. Synthesis of C-8 substituted quinolines. J. Heterocycl. Chem. 2001, 38, 233-236.

13. Mendez, L. Kouznetsov, V.; Poveda, J.; Yolaçan, C.; Ocal, N.; Aydogan, F. Transformations of 4- $\mathrm{N}$-arylamino-4-(8-quinolinyl)-1-butenes and 3-aryl-2-(8-quinolinyl)-4-thiazolidinones. Heterocycl. Commun. 2001, 7, 129-134.

14. Aydogan, F.; Ocal, N.; Turgut, Z.; Yolacan, C. Synthesis of thiazolidino-fused compounds. Bull. Korean Chem. Soc. 2001, 22, 476-480.

15. Shashikant, R.; Prajact, K.; Nachiket, S.; Sunil, A.; Deepak, S.; Smita, K.; Daithankar, A. Synthesis and biological evaluation of some 1, 3, 4-thiadiazoles. J. Chem. Pharm. Res. 2009, 1, 1191-1198.

16. Srivastava, S.D. Synthesis and Antimicrobial Activity of Some 2-[(4-Substituted-Phenyl-3-ChloroAzetidin-2-One)-5-(2'-Methylamino-4-Phenyl-1',3'-Thiazolyl-]-1,3,4-Thiadiazoles. J. Sci. Islam. Repub. Iran 2009, 20, 227-232.

17. Sharma, S.C. Synthesis of new fungicides. 2-(4'-arylthiazolyl-2'-imino)-3-aryl-4-thiazolidones. Bull. Chem. Soc. Jpn. 1967, 40, 2422-2424.

18. Akerblom, E.B. Synthesis and structureactivity relations of a series of antibacterially active 5-(5nitro-2-furfurylidene)thiazolones, 5-(5-nitro-2-urylpropenylidene)thiazolones, and 6-(5-nitro-2furyl)-4H-1,3-thiazinones. J. Med. Chem. 1974, 17, 609-615.

19. Frisch, M.J.; Trucks, G.W.; Schlegel, H.B.; Scuseria, G.E.; Robb, M.A.; Cheeseman, J.R.; Scalmani, G.; Barone, V.; Mennucci, B.; Petersson, G.A.; et al. Gaussian 09 series programsRevision A. 1; Gaussian, Inc.: Wallingford, CT, USA, 2009.

20. Lee, C.; Yang, W.; Parr, R. Synthesis and studying the antitumor activity of novel 5-(2-methylbenzimidazol-5-yl)-1,3,4-oxadiazole-2(3H)-thiones. Phys. Rev. B 1988, 37, 785-803.

21. Weigend, F.; Ahlrichs, R. Design and assessment of accuracy. Phys. Chem. Chem. Phys. 2005, 7, 3297-3304. 
22. Şahin, F.; Karaman, I.; Güllüce, M.; Öğütçü, H.; Şengül, M.; Adigüzel, A.; Öztürk, S.; Kotan, R. Evaluation of antimicrobial activities of Satureja hortensis L. J. Ethnopharmacol. 2003, 85, 231-238.

23. Soares, J.; Dins, T.; Cunha, A.; Ameida, L. Antioxidant activities of some extracts of thymus zygis. Free Radical Res. 1997, 26, 469-476.

24. Güven, K.; Yücel, E.; Çetintaş, F. Antimicrobial activities of fruits of crataegus and pyrus species. Pharm. Biol. 2006, 44, 79-86.

25. Tepe, B.; Daferera, D.; Sokmen, A.; Sokmen, M.; Polissiou, M. Antimicrobial and antioxidant activities of the essential oil and various extracts of Salvia tomentosa Miller (Lamiaceae). Food Chem. 2005, 90, 333-339.

Sample Availability: Samples of the compounds $\mathbf{2 - 5}$ are available from the authors.

(C) 2012 by the authors; licensee MDPI, Basel, Switzerland. This article is an open access article distributed under the terms and conditions of the Creative Commons Attribution license (http://creativecommons.org/licenses/by/3.0/). 